\title{
HYDROGEN AND HELIUM BURNING EVOLUTIONARY TRACKS
}

\author{
P.R. WOOD and E. VASSILIADIS \\ Mount Stromlo and Siding Spring Observatories, Private Bag, Weston Creek P.O., Canberra, \\ ACT 2611, Australia
}

\begin{abstract}
We discuss the effect of metallicity on the luminosities of planetary nebula nuclei (PNNi), and we use theoretical stellar evolution calculations to predict the fraction of PNNi that enter the planetary nebula domain burning helium rather than hydrogen. Both these factors will clearly influence the planetary nebula luminosity function .
\end{abstract}

\section{Introduction}

Stellar evolution calculations from the main sequence to the white dwarf stage have recently been made for stars of initial masses $1-5 \mathrm{M}_{\odot}$ and metal abundance $\mathrm{Z}$ from $\mathrm{Z}_{\odot}$ to $0.25 \mathrm{Z}_{\odot}$ (Vassiliadis and Wood 1992). These tracks allow us to predict the effect of metal abundance on the luminosities of PNNi produced by stars of a given initial mass. Of course, the luminosity of a PNN during its high luminosity transition across the HR diagram from the AGB is determined almost entirely by AGB mass loss. In these calculations, the empirical mass loss rate varies exponentially with pulsation period on the AGB up to a maximum value corresponding to the radiation pressure driven limit $\dot{M}=\mathrm{L} / \mathrm{cv}_{\infty}$ (Wood 1990; Vassiliadis and Wood 1992). There is no explicit abundance dependence in the mass loss formulae used, but the abundance is involved implicitly since lower abundance gives a hotter AGB which in turn leads to smaller pulsation periods at a given luminosity. Within the limits of current observational data, the maximum observed period for optically-visible, long-period variables on the AGB appears to be similar in the solar vicinity, the Galactic bulge, the LMC and the SMC. If we assume that a star will not be optically-visible once "superwind" mass loss starts, this result indicates that the high superwind mass loss rate begins at a similar pulsation period in each of these stellar systems, each of which has a different characteristic abundance. Furthermore, if we identify the superwind phase with the radiation pressure driven limit, then the result indicates that the mass loss rate depends mostly on the pulsation period, without further explicit abundance dependence. Our mass loss formula should therefore reasonably reproduce the transition to the superwind mass loss regime at different abundances. 
The second topic we wish to discuss is the likelihood that a star entering the planetary nebula region of the HR diagram will be burning helium or hydrogen. Since we have evolved a relatively large number of tracks (22) from the main-sequence to the PN domain without any deliberate attempt to control the phase of the shell flash cycle at which the stars leave the AGB, we can use the statistics of our $\mathrm{H}$ and $\mathrm{He}$ burning tracks to roughly estimate the relative numbers of observed PNNi that burn $\mathrm{H}$ or $\mathrm{He}$ on entering the PN domain of the HR diagram.

\section{Comparison of hydrogen and helium burning tracks}

The most detailed study of the possible PNN track morphologies that can result from stars leaving the AGB at different phases of the helium shell flash cycle is that of Iben (1984), this study being done for a $0.6 \mathrm{M}_{\odot}$ star. Basically, PNN tracks fall into two categories. Firstly, there are tracks where the star evolves smoothly through the PN region of the HR diagram burning hydrogen before the decline into the white dwarf state. Some examples of these tracks, which we will call hydrogen burning tracks, are shown in Figure 1. The second class of track is exhibited by stars that enter the PN domain of the HR

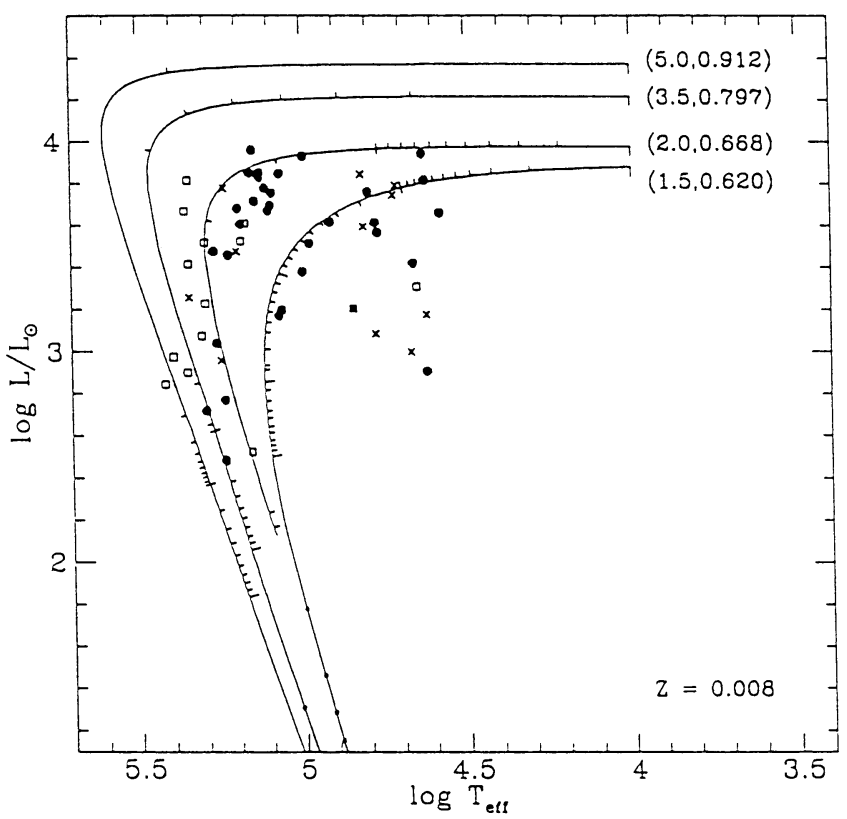

Figure 1. Evolutionary tracks for PNNi that cross the HR diagram burning hydrogen. Each track is labelled by (Initial mass, Mass at $\log \mathrm{T}_{\text {eff }}=4$ ). Tick marks are initially at intervals of $100 \mathrm{yr}$ (above track) then at $1000 \mathrm{yr}$ (below track). Dots mark time steps of $10^{5} \mathrm{yr}$ from $\log \mathrm{T}_{\text {eff }}=4$. Points represent LMC PN from Dopita and Meatheringham (1991a,b): open squares are type I PN, filled circles are optically thick objects, and crosses are optically thin objects. 


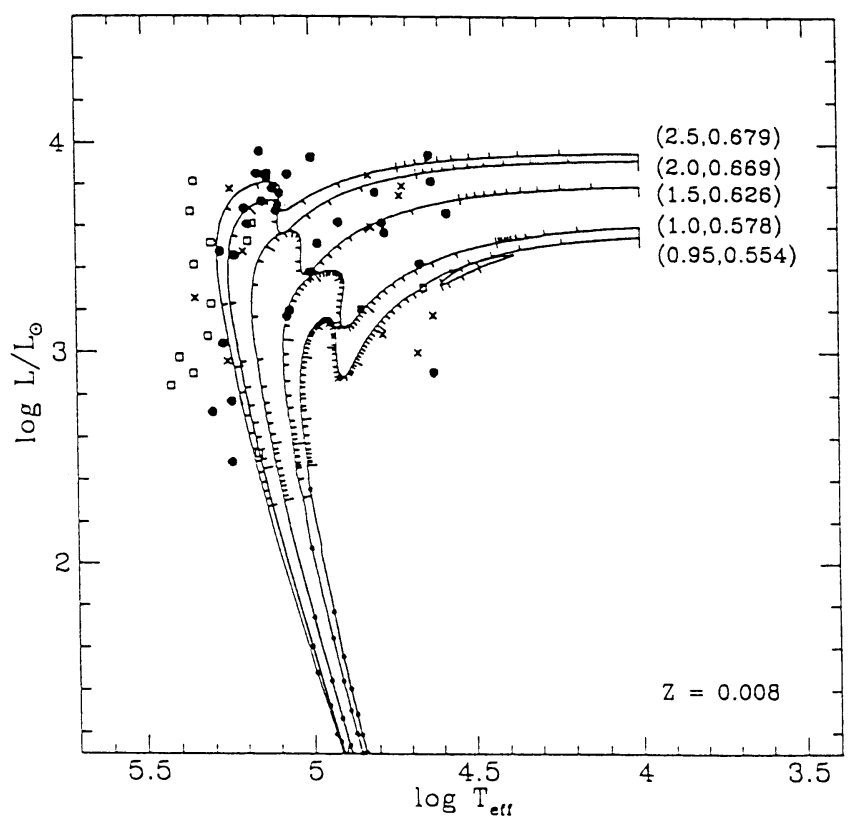

Figure 2. Same as Fig. 1 but for PNNi that enter the PN domain burning helium.

diagram burning helium (as a result of a helium shell flash) and which re-ignite the hydrogen-burning shell at high luminosity and high $\mathrm{T}_{\text {eff }}\left(\log \mathrm{T}_{\text {eff }} \approx 5\right)$. Such stars we will call helium burners, although this is a misnomer since the stars spend most of their PN lifetimes burning hydrogen. Examples of these tracks can be seen in Figure 2.

\section{The metal abundance dependence of PNN luminosity}

As can be seen in Figure 1, PNNi have their maximum luminosity when evolving across the HR diagram from the AGB. The luminosity $L(4)$ when $\log T_{\text {eff }}=4$ is given as a

\section{Table 1}

\begin{tabular}{cccccc}
\hline \hline $\mathrm{M}_{\mathrm{i}}$ & $\mathrm{Z}$ & $\log \mathrm{L}(4) / \mathrm{L}_{\odot}$ & $\mathrm{M}_{\mathrm{i}}$ & $\mathrm{Z}$ & $\log \mathrm{L}(4) / \mathrm{L}_{\odot}$ \\
\hline 1.0 & 0.016 & 3.541 & 2.5 & 0.016 & 3.979 \\
1.0 & 0.004 & 3.641 & 2.5 & 0.004 & 4.152 \\
1.0 & 0.001 & 3.776 & $\ldots$ & $\ldots$ & $\ldots$ \\
3.5 & 0.016 & 4.138 & 5.0 & 0.016 & 4.360 \\
3.5 & 0.008 & 4.216 & 5.0 & 0.008 & 4.370 \\
3.5 & 0.004 & 4.296 & 5.0 & 0.004 & 4.399 \\
\hline
\end{tabular}


function of initial mass $\mathrm{M}_{\mathrm{i}}$ in Table 1 for PNNi of various metal abundances. All tracks have initial helium abundance $\mathrm{Y}=0.25$.

It can be seen from Table 1 that $\operatorname{d} \log L(4) / \mathrm{d} \log \mathrm{Z} \sim-0.25$, at least for $\mathrm{M} \lesssim 3.5 \mathrm{M}_{\odot}$. The increase in maximum PNN luminosity with decreasing $\mathrm{Z}$ is as expected, since a higher metallicity gives a cooler giant branch and the period of $\sim 500$ days required for superwind mass loss rates occurs at lower luminosity.

\section{The fraction of hydrogen and helium burning PNNi}

Whether a star ends up as a hydrogen or helium burning PNN depends on (1) the phase of the shell flash cycle at which the star leaves the AGB (which in turn depends on how the mass loss rate varies throughout the shell flash cycle) and (2) the duration of the transition from the AGB to $\log \mathrm{T}_{\text {eff }}=4.5$ where nebula ionization becomes significant. Factors (1) and (2) together determine the phase of the shell flash cycle the star finds itself in when in the PN domain of the HR diagram.

In the present calculations, the very rapid increase of mass loss rate with luminosity means that, at least for the lower mass stars with $\mathrm{M} \lesssim 2.5_{\bullet}$, mass loss is concentrated in the latter half of the quiescent (hydrogen burning) phase of the shell flash cycle (Vassiliadis 1992; see also Wood 1992). If $\phi$ is the phase of the shell flash cycle (with the shell flash occurring at $\phi=0.0$ ), then the star is therefore most likely to leave the AGB during the interval $0.5<\phi<1.0$. Assuming a transition time of $\sim 7000$ years and a shell flash cycle length of $\sim 70000$ years, those PNN with $0.9 \leqslant \phi \lesssim 1.0$ will suffer a shell flash during post-AGB, pre-PN evolution and will therefore become helium burners (as defined above). In this case, we would expect $\sim 20 \%$ of low mass stars to become helium burning PNNi. In our calculations, we found that 6 out of 22 stars $(27 \%)$ became helium burners. If this factor is combined with the fact that the high luminosity phase of PN evolution is at least twice as long for helium burners as for hydrogen burners, then we would expect that at least $40 \%$ of observed high luminosity $\left(\log \mathrm{L} / \mathrm{L}_{\odot} \geq 2.5\right) \mathrm{PNNi}$ would be helium burners. This estimate is similar to that of Iben's (1984) guesses 1 and 2.

\section{References}

Blöcker, T. and Schönberner, D. 1990, A\&AL, 240, L11.

Iben, I. 1984, ApJ, 277, 333.

Paczynski, B. 1971, Acta Astr., 21, 417.

Vassiliadis, E. 1992, Thesis, Australian National University.

Vassiliadis, E. and Wood, P.R. 1992, in preparation.

Wood, P.R. 1990, in From Miras to Planetary Nebulae: Which Path for Stellar Evolution, eds. M.O. Mennessier and A. Omont (Editions Frontières), p.67.

Wood, P.R. 1992, in IAU Symposium 155 Planetary Nebulae, eds. R. Weinberger and A. Acker (Kluwer: Dordrecht), these proceedings.

Wood, P.R. and Faulkner, D.J. 1986, ApJ, 307, 659. 